\title{
Trispectrum versus bispectrum in single-field inflation
}

\author{
Kevin T. Engel, ${ }^{*}$ Keith S. M. Lee, ${ }^{\dagger}$ and Mark B. Wise ${ }^{\ddagger}$ \\ California Institute of Technology, Pasadena, California 91125, USA \\ (Received 23 January 2009; published 28 May 2009; publisher error corrected 16 June 2009)
}

\begin{abstract}
In the standard slow-roll inflationary cosmology, quantum fluctuations in a single field, the inflaton, generate approximately Gaussian primordial density perturbations. At present, the bispectrum and trispectrum of the density perturbations have not been observed and the probability distribution for these perturbations is consistent with Gaussianity. However, Planck satellite data will bring a new level of precision to bear on this issue, and it is possible that evidence for non-Gaussian effects in the primordial distribution will be discovered. One possibility is that a trispectrum will be observed without evidence for a nonzero bispectrum. It is not difficult for this to occur in inflationary models where quantum fluctuations in a field other than the inflaton contribute to the density perturbations. A natural question to ask is whether such an observation would rule out the standard scenarios. We explore this issue and find that it is possible to construct single-field models in which inflaton-generated primordial density perturbations have an observable trispectrum, but a bispectrum that is too small to be observed by the Planck satellite. However, an awkward fine-tuning seems to be unavoidable.
\end{abstract}

DOI: 10.1103/PhysRevD.79.103530

PACS numbers: $98.80 . \mathrm{Cq}$

\section{INTRODUCTION}

Inflationary cosmology is a scenario that solves the horizon and flatness problems [1-3]. Furthermore, it provides a method for generating the approximately scaleinvariant primordial density perturbations that are responsible for the large-scale structure in the universe, as well as the anisotropy of the cosmic microwave background radiation [4-6]. Although inflation has become the standard paradigm for early-universe cosmology, there is no direct evidence that supports this paradigm, since it occurs at a very high energy scale. In the standard slow-roll inflationary cosmology a single field, the inflaton, is responsible for inflation and generates the primordial density perturbations. In this case, the density perturbations are approximately Gaussian $[7,8]$. However, other mechanisms for generating the density perturbations can give rise to significant non-Gaussian effects [9]. For example, DiracBorn-Infeld (DBI) inflation and its generalizations, in which there is a small speed of sound, $c_{s}$, during inflation, give rise to a large bispectrum, and the present limits from WMAP already constrain such models [10-13].

The Planck satellite will take us to a new level of precision in the measurement of the anisotropy of the microwave background radiation, and it is possible that non-Gaussianities will be observed [14,15]. In particle physics, it is not unusual to consider a scalar field theory that has a connected four-point correlation but no threepoint correlation. For example, if a $\phi \rightarrow-\phi$ symmetry is imposed then the three-point correlation vanishes. This is essentially the same mechanism that causes the model of

\footnotetext{
*kte@caltech.edu

†ksml@caltech.edu

¥wise@ theory.caltech.edu
}

Ref. [16] to have a significant trispectrum but no bispectrum. The same signature of non-Gaussianities can also appear in the curvaton model if some cancellation of terms occurs [17-19].

A natural question that arises within the inflationary paradigm is whether the observation of a trispectrum for the primordial density perturbations but no bispectrum would imply that there must be more than one scalar field playing a role in inflation. In this paper, we explore whether models with a single scalar field that is responsible for both inflation and the generation of the density perturbations can give rise to such a signature in the Planck data. We find that this is possible, but that it seems to require a fine-tuning of parameters. Our work shows that such an observation would not rule out single-field models but, because of this fine-tuning, we would view these models as disfavored.

The rest of this paper is organized as follows. In Sec. II, we briefly review the formalism used for general inflationary models. In Sec. III, we discuss non-Gaussianities in primordial density perturbations. Some general models that feature a large trispectrum but small bispectrum are constructed in Sec. IV. Then, in Sec. V, we consider specific examples and perform numerical studies of their behavior, in particular, investigating issues of fine-tuning. We conclude in Sec. VI.

\section{FORMALISM}

Consider a general Lagrangian density for the inflaton of the form $\mathcal{L}=\sqrt{-g} P(X, \phi)$, where $X=-\frac{1}{2} g^{\mu \nu} \partial_{\mu} \phi \partial_{\nu} \phi$. Here, $P$ plays the role of pressure. Such a setup was studied in Ref. [13], where it was applied to kinetically driven inflation (referred to as " $k$-inflation"). The background solution for the inflaton is taken to be spatially homoge- 
neous, $\phi=\phi(t)$. Then, $X=\frac{1}{2} \dot{\phi}^{2} . P(X, \phi)$ should satisfy the following requirements:

$$
\begin{gathered}
\text { (i) } \frac{\partial P}{\partial X} \geq 0, \\
\text { (ii) } X \frac{\partial P}{\partial X}-P \geq 0, \\
\text { (iii) } 2 X \frac{\partial^{2} P}{\partial X^{2}}+\frac{\partial P}{\partial X}>0 .
\end{gathered}
$$

The first two requirements are implied by the dominant energy condition, while the last condition (see e.g. Ref. [20]) ensures that the theory is well defined.

The evolution of the universe is governed by the Friedmann and continuity equations,

$$
H^{2}=\frac{1}{3 M_{P}^{2}} \rho, \quad \dot{\rho}=-3 H(\rho+P),
$$

where $H$ is the Hubble parameter, $M_{P}=(8 \pi G)^{-1 / 2}$ is the reduced Planck mass, and the energy density, $\rho$, is given by

$$
\rho=2 X \frac{\partial P}{\partial X}-P .
$$

The familiar case of slow-roll inflation utilizes a flat potential, whose slope and curvature are characterized by the slow-roll parameters $\epsilon, \eta \ll 1$. In general, however, the potential may be relatively steep. An example is provided by DBI inflation, in which the inflaton $\phi$ corresponds to the position of a D3-brane rolling down a warped throat. The Lagrangian is of the form

$$
P=-f(\phi)^{-1}[\sqrt{1-2 X f(\phi)}-1]-V(\phi),
$$

and the warping results in a speed limit of $X \rightarrow 1 /(2 f(\phi))$.

The slow-roll parameters are then generalized to

$$
\begin{gathered}
\epsilon \equiv-\frac{\dot{H}}{H^{2}}=\frac{3 X \frac{\partial P}{\partial X}}{2 X \frac{\partial P}{\partial X}-P}, \\
\eta \equiv \frac{\dot{\epsilon}}{\epsilon H}, \\
s \equiv \frac{\dot{c}_{s}}{c_{s} H},
\end{gathered}
$$

where the "speed of sound," $c_{s}$, is given by

$$
c_{s}^{2}=\frac{\frac{\partial P}{\partial X}}{\frac{\partial P}{\partial X}+2 X \frac{\partial^{2} P}{\partial X^{2}}} .
$$

In models with a standard kinetic term, $c_{s}=1$ and $s=0$. In contrast, $c_{s} \ll 1$ is possible in DBI inflation; this leads to significant non-Gaussianities, as will be described in the following section.
The running of the power spectrum is parametrized by the spectral index, $n_{s}$, which is related to the generalized slow-roll parameters by

$$
n_{s}-1=-2 \epsilon-\eta-s .
$$

Since WMAP observes an almost scale-invariant power spectrum, the three slow-variation parameters are constrained to be of order $10^{-2}$.

\section{NON-GAUSSIANITIES}

It is anticipated that the Planck satellite will provide sufficiently precise data for it to be possible to begin distinguishing between various inflationary models. One would like to be able to rule out, or at least disfavor, broad classes of models. (A formalism for reconstructing a general single-field action from phenomenological inputs is given in Ref. [21].) In this endeavour, several observables will play a critical role, namely, the spectral index, tensor perturbations (primordial gravity waves), and nonGaussianity. If the density perturbations are Gaussian, with uncorrelated Fourier modes, then the two-point correlation function fully characterizes the distribution. In particular, all odd correlation functions are zero, while higher-order even correlation functions can be expressed in terms of the two-point function. For example, the fourpoint function of a Gaussian variable $\zeta$ is $\left\langle\zeta_{1} \zeta_{2} \zeta_{3} \zeta_{4}\right\rangle=$ $\left\langle\zeta_{1} \zeta_{2}\right\rangle\left\langle\zeta_{3} \zeta_{4}\right\rangle+\left\langle\zeta_{1} \zeta_{3}\right\rangle\left\langle\zeta_{2} \zeta_{4}\right\rangle+\left\langle\zeta_{1} \zeta_{4}\right\rangle\left\langle\zeta_{2} \zeta_{3}\right\rangle$. Thus, the detection of a three-point function or a "connected" part of the four-point function (or, equivalently, their Fourier transforms, the bispectrum and trispectrum, respectively) would signal non-Gaussianities. Correlations of this kind are predicted to be undetectably small in slow-roll models of inflation, but can be large in models with a nonstandard kinetic term, such as DBI inflation.

It is convenient to characterize the size of the bispectrum by the parameter $f_{\mathrm{NL}}$, given by [14]

$$
\begin{aligned}
\left\langle\zeta\left(\mathbf{k}_{1}\right) \zeta\left(\mathbf{k}_{2}\right)\right\rangle & =(2 \pi)^{3} \delta^{3}\left(\mathbf{k}_{1}+\mathbf{k}_{2}\right) P_{\zeta}(k), \\
\left\langle\zeta\left(\mathbf{k}_{1}\right) \zeta\left(\mathbf{k}_{2}\right) \zeta\left(\mathbf{k}_{3}\right)\right\rangle= & -(2 \pi)^{3} \delta^{3}\left(\mathbf{k}_{1}+\mathbf{k}_{2}+\mathbf{k}_{3}\right) \\
& \times \frac{6}{5} f_{\mathrm{NL}}\left[P_{\zeta}\left(k_{1}\right) P_{\zeta}\left(k_{2}\right)+\text { perms }\right],
\end{aligned}
$$

where $\zeta$ is the gauge-invariant curvature perturbation and "perms" stands for two permutations of the indices. The general expression for the power spectrum appearing in the formulae above is [13]

$$
P_{\zeta}(k)=\frac{1}{4 M_{P}^{2}} \frac{1}{k^{3}} \frac{H^{2}}{c_{s} \epsilon} .
$$

In general, $f_{\mathrm{NL}}$ will be a function of the wave numbers $\mathbf{k}_{i}$ [7]. In practice, a comparison with data will involve evaluating the bispectrum for a particular configuration of the $\mathbf{k}_{i}$, conventionally the "equilateral" configuration, in which $k_{i}=k$. Then one can set up an estimator for bispectra that have momentum dependences different from that of 
the "local" non-Gaussianity, where $f_{\mathrm{NL}}$ is a constant $[22,23]$.

Likewise, the size of the trispectrum is conveniently characterized by the parameter $\tau_{\mathrm{NL}}$, which is given by [24]

$$
\begin{aligned}
\left\langle\zeta\left(\mathbf{k}_{1}\right) \zeta\left(\mathbf{k}_{2}\right) \zeta\left(\mathbf{k}_{3}\right) \zeta\left(\mathbf{k}_{\mathbf{4}}\right)\right\rangle_{\mathrm{c}}= & (2 \pi)^{3} \delta^{3}\left(\mathbf{k}_{1}+\mathbf{k}_{2}+\mathbf{k}_{3}+\mathbf{k}_{4}\right) \\
& \times \frac{1}{2} \tau_{\mathrm{NL}}\left[P_{\zeta}\left(k_{1}\right) P_{\zeta}\left(k_{2}\right) P_{\zeta}\left(k_{14}\right)\right. \\
& + \text { perms }],
\end{aligned}
$$

where the subscript "c" indicates the connected part, $k_{14}=\left|\mathbf{k}_{1}+\mathbf{k}_{4}\right|$, and "perms" stands for 23 permutations of the indices. (In fact, half of the permutations are equal to the other half, as a consequence of the condition $\sum_{i} \mathbf{k}_{i}=$ 0 .) In general, $\tau_{\mathrm{NL}}$ will depend upon the $\mathbf{k}_{i}$ and, unlike $f_{\mathrm{NL}}$, even in the equilateral configuration will still have angular dependence (and is not restricted to lie in a plane). One can then choose a particular angular configuration, by specifying the three angles $\cos \theta_{m 4}, m=1,2,3$, where $\theta_{i j}$ is the angle between $\mathbf{k}_{i}$ and $\mathbf{k}_{j}$. The current bounds from WMAP and COBE are $-4<f_{\mathrm{NL}}^{\text {local }}<80[25],-151<f_{\mathrm{NL}}^{\text {equil }}<$ 253 [26], and $\left|\tau_{\mathrm{NL}}^{\text {local }}\right| \lesssim 10^{8}$ [24], but Planck is expected to achieve a sensitivity down to $\left|f_{\mathrm{NL}}^{\text {local }}\right| \approx 5[14],\left|f_{\mathrm{NL}}^{\text {equil }}\right| \approx$ $66($ at $1 \sigma)[27,28]$, and $\left|\tau_{\mathrm{NL}}^{\text {local }}\right| \approx 560$ [15].

The general expression for $f_{\mathrm{NL}}$ was derived in Ref. [29], which built upon work in Ref. [30]. The momentum dependence of the three-point function was decomposed into six functions, four of which are suppressed by slow-roll parameters. In the equilateral configuration, the two remaining contributions give

$$
f_{\mathrm{NL}}^{c}=\frac{35}{108}\left(\frac{1}{c_{s}^{2}}-1\right),
$$

and

$$
f_{\mathrm{NL}}^{\lambda}=-\frac{5}{81}\left[\left(\frac{1}{c_{s}^{2}}-1-\frac{2 \lambda}{\Sigma}\right)+\left(3-2 \mathbf{c}_{1}\right) \frac{l \lambda}{\Sigma}\right]
$$

where $\mathbf{c}_{1}=0.5772 \ldots$ is the Euler-Mascheroni constant and

$$
\lambda \equiv X^{2} P_{, X X}+\frac{2}{3} X^{3} P_{, X X X}, \quad \Sigma \equiv X P_{, X}+2 X^{2} P_{, X X} .
$$

Here, $l \equiv \dot{\lambda} /(\lambda H)$ is a fourth slow-variation parameter, and we have adopted the notation $P_{,_{X}}=\partial P / \partial X$, etc. The formulae above make it clear that a large $f_{\mathrm{NL}}$ requires either $c_{s}^{2} \ll 1$ or $\lambda / \Sigma \gg 1$. For standard slow-roll models, $f_{\mathrm{NL}}$ is negligible, since $c_{s}^{2}=1$ and $\lambda=0$. DBI inflation, on the other hand, predicts a large bispectrum, with both $c_{s}^{2} \ll 1$ and $\lambda / \Sigma \gg 1$. In this particular case, $f_{\mathrm{NL}}^{c}$ gives the dominant contribution, as the first three terms in Eq. (17) cancel, so that $f_{\mathrm{NL}}^{\lambda}$ is suppressed by a factor of $l$.

A model with an unobservable bispectrum, then, must have $c_{s}^{2} \approx 1$ and $\lambda / \Sigma \lesssim \mathcal{O}(1)$. In this limit, the only potentially sizeable contribution to $\tau_{\mathrm{NL}}$ comes from a term analogous to the $\lambda / \Sigma$ term in $f_{\mathrm{NL}}$. Just as $f_{\mathrm{NL}}$ can be large if $P_{, X X X} \gg 1, \tau_{\mathrm{NL}}$ can be large if $P_{, X X X X} \gg 1$. This leading piece can be determined from Eqs. (14) and (15) and the trispectrum calculations in Refs. [31,32]. We obtain

$$
\begin{aligned}
\tau_{\mathrm{NL}}\left[\frac{1}{k_{1}^{3}} \frac{1}{k_{2}^{3}} \frac{1}{k_{14}^{3}}+\text { perms }\right]= & 384 \frac{1}{k_{1} k_{2} k_{3} k_{4}} \frac{1}{K^{5}} X^{4} P_{, X X X X} \\
& \times \frac{c_{s}^{2}}{M_{P}^{2} H^{2} \epsilon}+\cdots
\end{aligned}
$$

where $K=\sum_{i} k_{i}$. For the equilateral configuration, in which $k_{i}=k$ and $\sum_{m=1}^{3} \cos \theta_{m 4}=-1$, Eq. (19) becomes

$$
\begin{aligned}
\tau_{\mathrm{NL}}= & \frac{3 \sqrt{2}}{32} \frac{1}{\sum_{m=1}^{3}\left(1+\cos \theta_{m 4}\right)^{-3 / 2}} X^{4} P_{, X X X X} \frac{c_{s}^{2}}{M_{P}^{2} H^{2} \epsilon} \\
& +\cdots .
\end{aligned}
$$

Finally, choosing the configuration $\cos \theta_{m 4}=-1 / 3$, which maximizes Eq. (20), we obtain

$$
\tau_{\mathrm{NL}}=\frac{\sqrt{3}}{72} X^{4} P_{, X X X X} \frac{c_{s}^{2}}{M_{P}^{2} H^{2} \epsilon}+\cdots,
$$

which, combined with Eqs. (7) and (10), gives

$$
\tau_{\mathrm{NL}}=\frac{\sqrt{3}}{72} \frac{X^{4} P_{, X X X X}}{X P_{, X}+2 X^{2} P_{, X X}}+\cdots .
$$

(Recall that $c_{s}^{2} \approx 1$ in Eqs. (19)-(22).)

In many inflationary models, the magnitudes of $f_{\mathrm{NL}}$ and $\tau_{\mathrm{NL}}$ are either both small or both large. In slow-roll models, for example, $\tau_{\mathrm{NL}} \sim f_{\mathrm{NL}} \sim \epsilon[33,34]$, whereas in DBI inflation we find that (see Eq. (17) and Ref. [32])

$$
\tau_{\mathrm{NL}}^{\mathrm{DBI}}=-\frac{729}{1225 \sqrt{3}} f_{\mathrm{NL}}^{2}+\cdots .
$$

In the following section, we address the question of whether it is possible to construct a single-field model that has a large trispectrum but small bispectrum. Such a non-Gaussian signature is possible in the curvaton model, ${ }^{1}$ provided that it has a self-interaction term in its potential. In this scenario, the inflaton drives inflation as usual, but a separate scalar field, known as the curvaton, produces the curvature perturbations.

In order to have $\tau_{\mathrm{NL}} \gg f_{\mathrm{NL}}$, Eqs. (17) and (22) indicate that one should have $P_{, X X X X} \gg P_{, X X X}$. This will occur if $\dot{\phi}$ converges to a speed limit, at which the fourth derivative is singular while the third derivative is nonsingular (or mildly singular). Generically, such a model will involve a finetuning, i.e. there will be a relationship between the potential, $V$, and other terms in the Lagrangian, as can be seen by

\footnotetext{
${ }^{1}$ In this case, the trispectrum is described by two parameters, $\tau_{\mathrm{NL}}$ and $g_{\mathrm{NL}}$, and it is the latter that can be very large. Of course, consideration of a specific configuration effectively discards such distinctions between different shapes (momentum dependences).
} 
simply substituting the speed limit into the equation of motion. DBI inflation, however, is an exception: the speed limit occurs without imposing any restriction on $V$. This is because even the first derivative has a nonanalyticity; hence, in the equation of motion, as $1 / \sqrt{1-f \dot{\phi}^{2}} \rightarrow \infty$ the terms involving $V$ vanish. If, on the other hand, neither the first nor the second derivative blows up, then there is no divergence in the equation of motion.

\section{MODEL BUILDING}

We are interested in constructing a model with $P_{, X X X X}$ parametrically larger than $P_{, X X X}$. One obvious solution is obtained by taking the DBI kinetic term to a higher power:

$$
P(X, \phi) \sim f(\phi)^{-1}(1-f(\phi) X)^{\alpha} .
$$

For $3<\alpha<4$, as $X \rightarrow f^{-1}$ the fourth derivative becomes singular while the lower derivatives tend asymptotically to 0 . However, if this is the only kinetic term, the theory predicts a large $f_{\mathrm{NL}}$. Applying Eq. (10) to our model, we find that in the limit $X \rightarrow f^{-1}$

$$
c_{s}^{2} \sim \frac{1-f X}{2(1-\alpha)} \rightarrow 0 .
$$

Because small $c_{s}^{2}$ corresponds to large $f_{\mathrm{NL}}$, this must be avoided. The simplest remedy is to insert a standard kinetic term. With the addition of functional coefficients and a potential, our first model (referred to henceforth as the " $\alpha$ model") is given by

$$
P(X, \phi)=A X+B f^{-1}(1-f X)^{\alpha}-U,
$$

where $A, B, f$, and $U$ can all be functions of $\phi$, and $U=$ $V+B f^{-1}$. A few restrictions can be imposed on this theory from the beginning. First, for small $X$, the second term can be expanded, generating the standard canonical kinetic term if we require

$$
\left.(A-\alpha B)\right|_{\phi=0}=1 .
$$

Another constraint is given by the energy density, which can be calculated from Eq. (5):

$$
\rho=A X-B(1-f X)^{\alpha-1}\left[(2 \alpha-1) X+f^{-1}\right]+U .
$$

Unlike DBI inflation, this theory contains no singular terms in its equation of motion; hence $X$ could potentially surpass $f^{-1}$. In order to avoid imaginary terms in the energy density, $\alpha$ must therefore have an odd denominator.

With these restrictions in place, a given set of parameters produces a sensible theory where $\phi$ rolls to the minimum of the potential. $\tau_{\mathrm{NL}}$ becomes large, however, only when $\frac{1}{2} \dot{\phi}^{2}$ is close to $f^{-1}$. In order to produce large nonGaussianities from generic initial conditions, we must therefore impose a speed limit. We do this by inserting our desired speed limit into the equation of motion for $\phi$ :

$$
X=\frac{1}{2} \dot{\phi}^{2} \rightarrow \frac{1}{f}, \quad \ddot{\phi} \rightarrow \frac{-f^{\prime}}{f^{2}},
$$

where the prime denotes a derivative with respect to $\phi$. With these insertions several terms drop out, and the remainder can be integrated to give

$$
\left(U+\frac{A}{f}\right)^{1 / 2}=\frac{\sqrt{6}}{2 M_{P}} \int \frac{A}{\sqrt{f}} d \phi .
$$

To obtain a speed limit, we are forced to have this relationship between the kinetic and potential terms. For a given $A, f$, and $B, V$ is nearly completely specified (some minimal freedom in lower-order terms exists due to the constant of integration).

We can check the stability of this solution by expanding around the speed limit, writing $X \rightarrow \frac{1}{f}(1-\varepsilon)$. For small $\varepsilon$, we find that

$$
\frac{\dot{\varepsilon}}{\varepsilon}=\sqrt{\frac{2}{f}}\left[\frac{A^{\prime}}{A}-\frac{f^{\prime}}{f}\right]-\frac{\sqrt{3}}{M_{P}}\left[\left(\frac{A}{f}+U\right)^{1 / 2}+\frac{A}{f\left(\frac{A}{f}+U\right)^{1 / 2}}\right] .
$$

For the models we consider, the negative second term is dominant, thereby driving $\varepsilon$ to 0 . With $\dot{\phi}$ forced to the speed limit, the non-Gaussianities are straightforward to calculate. In the equilateral configuration, the dominant contribution to $\tau_{\mathrm{NL}}$ is given by

$$
\tau_{\mathrm{NL}}=\frac{\sqrt{3}}{72} \frac{B}{A} \alpha(\alpha-1)(\alpha-2)(\alpha-3) \varepsilon^{\alpha-4} .
$$

The main contribution to $f_{\mathrm{NL}}$, on the other hand, is given by

$$
f_{\mathrm{NL}} \sim \frac{B}{A} \varepsilon^{\alpha-3} .
$$

As the inflaton rolls to its speed limit, a suitable choice for $\alpha$ renders $f_{\mathrm{NL}}$ small and $\tau_{\mathrm{NL}}$ large. We have achieved our goal, encoding the non-Gaussianities for this model in the trispectrum rather than the bispectrum, but to obtain this result we have had to tune the potential. We can examine the degree of fine-tuning required by perturbing the potential. Making the replacements

$$
X \rightarrow \frac{1}{f}(1-\varepsilon), \quad V \rightarrow V+\delta V,
$$

we expand to first order in $\varepsilon$ and $\delta V$ and ignore $\dot{\varepsilon}$ terms. The result is an equation for $\varepsilon$ :

$$
\varepsilon=\frac{f \delta V-\frac{2 M_{P}}{\sqrt{6}} \frac{(A+f U)^{1 / 2}}{A} f \delta V^{\prime}}{2 A+f U+\frac{2 M_{P}}{\sqrt{6}}(A+f U)^{1 / 2}\left(\frac{f^{\prime}}{f}-\frac{A^{\prime}}{A}\right)} .
$$

For unperturbed $V$, Eq. (31) shows that $\varepsilon$ decreases roughly exponentially. With perturbations, however, $\varepsilon$ tends to level off at some nonzero value. We shall show with specific examples in the next section that for large enough 
perturbations $\varepsilon$ does not become small enough to generate an observable $\tau_{\mathrm{NL}}$.

From a tuning standpoint, then, we are interested in enhancing $\tau_{\mathrm{NL}}$ so that $\varepsilon$ does not have to be extremely small, thus allowing a wider range of potentials. From Eq. (32), we see that a large value for $B / A$ appears to accomplish this goal. However, for generic initial conditions not at the speed limit, the ratio is bounded. The first dominant energy condition (Eq. (1)) evaluated at $\dot{\phi}=0$ gives

$$
A-\alpha B \geq 0 .
$$

The same equation evaluated at the speed limit implies that $A$ must be positive; therefore, for positive $B, B / A \leq 1 / \alpha$. For negative $B$, the restriction appears in the equation of motion. For large $|B / A|$ the coefficient of $\ddot{\phi}$ can evolve to 0 , causing the equation to become singular, unless

$$
\left|\frac{B}{A}\right|<\frac{1}{2 \alpha}\left(1-\frac{3}{2 \alpha-1}\right)^{2-\alpha} .
$$

These two conditions force us to impose

$$
\left|\frac{B}{A}\right| \lesssim \frac{1}{\alpha} \quad \text { (before hitting speed limit). }
$$

Once $\phi$ is at the speed limit, however, $B$ vanishes from the equation of motion and can be set arbitrarily large. For clever choices of the functions $A$ and $B, B / A$ can be made small initially, but larger later on, thus enhancing $\tau_{\mathrm{NL}}$. Despite its apparent promise, this idea actually has limited utility, for a couple of reasons. First, the requirement of a canonical kinetic term forces the use of unappealing functional forms: the simplest model we found had $B$ as a Gaussian in $\phi$. More importantly, $f_{\mathrm{NL}}$ also scales with $B / A$, which limits the overall use of the ratio to magnify $\tau_{\mathrm{NL}}$.

With the constraint that $B / A$ is of order unity, the last way to enhance $\tau_{\mathrm{NL}}$ is to choose an $\alpha$ as small as possible. This leads to a preferred $\alpha$ for each of the allowed odd denominators:

$$
\alpha=\frac{10}{3}, \frac{16}{5}, \frac{22}{7}, \ldots
$$

Considering the $\varepsilon$ dependence of $\tau_{\mathrm{NL}}$ at the end point of this series leads us to our second model, designated the "log model":

$$
P(X, \phi)=A X+B f^{-1}(1-f X)^{3} \log |1-f X|-U,
$$

where in this model $U=V$. Most of the equations from the previous model carry over. In particular, the potential equation (30), the stability equation (31), and the perturbation equation (35) all continue to hold for the log model. The canonical constraint changes to

$$
\left.(A-B)\right|_{\phi=0}=1,
$$

and a similar analysis to the one above shows that

$$
\left|\frac{B}{A}\right| \lesssim 1 \quad \text { (before hitting speed limit). }
$$

The main difference between the two models is the signature of the non-Gaussianities. In the equilateral configuration, to leading order we have

$$
\begin{gathered}
f_{\mathrm{NL}}=-\frac{40}{81} \frac{B}{A} \log |\varepsilon|, \\
\tau_{\mathrm{NL}}=\frac{\sqrt{3}}{12} \frac{B}{A} \varepsilon^{-1} .
\end{gathered}
$$

We see that $f_{\mathrm{NL}}$ now diverges as $\varepsilon \rightarrow 0$, albeit more slowly than $\tau_{\mathrm{NL}}$. Also, for the same $\varepsilon, \tau_{\mathrm{NL}}$ is larger in the $\log$ model than in the $\alpha$ model, which in turn allows a less finetuned potential. We shall explore these models and the requisite fine-tuning with numerical simulation of specific examples in the next section.

\section{NUMERICAL STUDIES}

We consider numerical solutions to the equation of motion using initial conditions $\phi(0)=\phi_{0}$ and $\dot{\phi}(0)=0$. An initial velocity for $\phi$ makes little difference to the end result as $\phi$ is generally driven quickly to the speed limit. The initial value for $\phi$ is set by the need for a nearly scaleinvariant spectrum. The slow-variation parameter $\epsilon$ is required to be $\mathcal{O}\left(10^{-2}\right)$, and this usually imposes a lower bound on $\phi$. Two other observational constraints that need to be considered are the amplitude of the power spectrum and the number of e-foldings. For single-field inflation the size of the density perturbations is characterized by

$$
\Delta_{\mathcal{R}}^{2}=\frac{k^{3} P_{\zeta}}{2 \pi^{2}}=\frac{H^{2}}{8 \pi^{2} M_{P}^{2} c_{s} \epsilon},
$$

whose value has been observed to be approximately $2.45 \times$ $10^{-9}$ [26]. The effect of this constraint in our models is to set a mass scale. The relevant equation for the number of efoldings is

$$
N_{e}=\int_{\phi_{i}}^{\phi_{f}} \frac{H}{\dot{\phi}} d \phi .
$$

Cosmological scales of interest exit the horizon 50-60 efolds before the end of inflation. We need to ensure that there is a sufficiently long observation window, during which $\tau_{\mathrm{NL}}$ should be large, and also that roughly 60 e-folds can be attained in total. Like $\epsilon$, this puts a lower bound on $\phi_{0}$. Finally, one other observable of interest is $r$, the tensorto-scalar ratio. Garriga and Mukhanov derived in Ref. [13] the result that for general single-field models of inflation

$$
r=16 c_{s} \epsilon
$$

For the models we consider here, $c_{s}=1$. To obtain $n_{s}$ 
consistent with observations, $\epsilon \sim .01$; therefore, our models predict a value for $r$ in the range $0.1-0.2$.

\section{A. $\alpha$ model}

The simplest model is the one in which $A, B$, and $f$ are all constant: for example,

$$
A=2, \quad B=\frac{1}{\alpha}, \quad f=\frac{6}{m^{4}}, \quad \alpha=\frac{10}{3} .
$$

The potential is then given by Eq. (30):

$$
V=m^{4}\left[\left(\frac{\phi}{M_{P}}\right)^{2}-\frac{1}{3}-\frac{1}{6 \alpha}\right] .
$$

The negative energy density that can result for small $\phi$ is not a concern here, for it turns out that inflation occurs only for $\phi>M_{P}$. The inflation era ends when $\ddot{a} \leq 0$ or equivalently $\epsilon \geq 1$. Equation (7) evaluated at the speed limit gives

$$
\epsilon=\frac{M_{P}^{2}}{\phi^{2}} .
$$

We see that $\phi$ must be larger than $1 M_{P}$ during inflation and that $\phi \sim 10 M_{P}$ as current cosmological scales were exiting the horizon. The number of e-folds, calculated to be

$$
N_{e}=\frac{1}{2}\left[\left(\frac{\phi_{i}}{M_{P}}\right)^{2}-\left(\frac{\phi_{f}}{M_{P}}\right)^{2}\right],
$$

imposes a similar restriction on $\phi$. In order to achieve the final $60 \mathrm{e}$-folds, the end of the observation window must be at or beyond $\phi=11 M_{P}$. Finally, we can calculate $\Delta_{\mathcal{R}}^{2}$ from Eq. (43):

$$
\Delta_{\mathcal{R}}^{2}=\frac{1}{24 \pi^{2}}\left(\frac{m}{M_{P}}\right)^{4}\left(\frac{\phi}{M_{P}}\right)^{4}=2.45 \times 10^{-9} .
$$

Substituting in $\phi=12 M_{P}$, we find that $m \sim 2 \times 10^{-3} M_{P}$.

For the initial conditions $\phi(0)=13 M_{P}$, and $\dot{\phi}(0)=0$, the key features of the evolution are shown in Fig. 1. As predicted, $\tau_{\mathrm{NL}}$ grows roughly exponentially for $V$ that satisfies Eq. (30) exactly. Since $\left|\tau_{\mathrm{NL}}\right|$ has been observationally constrained to be less than $10^{8}$, this point signifies the end of our possible observational window. To enforce this, we require some exit mechanism to kick in around $\phi=$ $5 M_{P}$, ending inflation. The beginning of our observational window is set by $n_{s}$, which becomes flat only as $\dot{\phi}$ approaches the speed limit. For this model, the observational window occurs for $\phi$ in the range $12.85 M_{P}>\phi>$ $12.10 M_{P}$, lasting about 9 e-folds. This window corresponds to the range of observable wave numbers, $k . f_{\mathrm{NL}}$ is unobservably small throughout, but $\tau_{\mathrm{NL}}$ is very $k$ dependent, growing from $\sim 1$ at large scales to $10^{8}$ at the smallest scales.

These results are not typical ones, however: an inflaton potential even slightly modified from one satisfying Eq. (30) exactly will lead to a significantly different signature. We investigate the allowed sizes of these deviations and their effects on $\tau_{\mathrm{NL}}$ by perturbing the coefficients in the potential. Figure 2 shows the result of perturbing the mass term by

$$
\delta V=m^{4} \delta\left(\frac{\phi}{M_{P}}\right)^{2}
$$

for several different values of $\delta$. Altering other coefficients gives similar results; therefore, the general effect of poten-

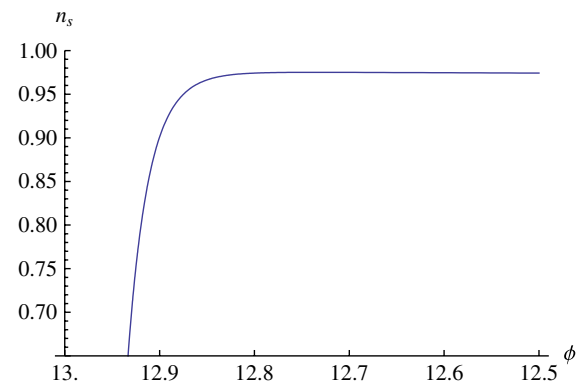

(b) (a)

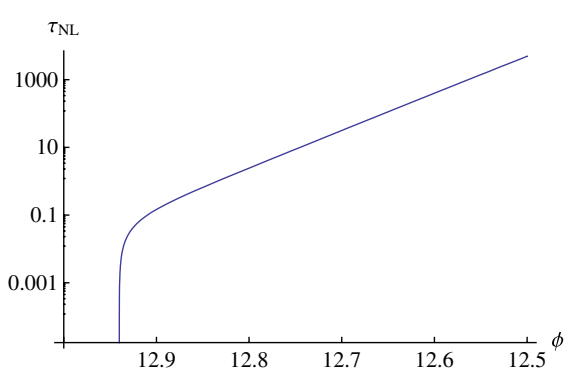

(c)

FIG. 1 (color online). (a) $\dot{\phi}$ (solid line) plotted with speed limit of $-\sqrt{\frac{2}{f}}$ (dashed line). (b) Spectral index $n_{s}$. (c) $\tau_{\mathrm{NL}}$. 


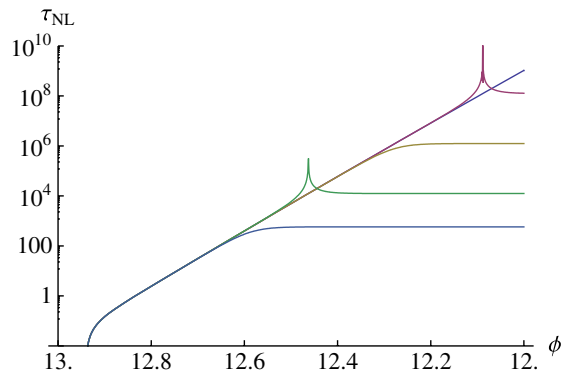

FIG. 2 (color online). $\tau_{\mathrm{NL}}$ plotted for various values of $\delta$. From top to bottom, $\delta=0,10^{-15},-10^{-12}, 10^{-9},-10^{-7}$. For positive $\delta, \dot{\phi}$ crosses the speed limit before leveling off, creating the spikes shown in $\tau_{\mathrm{NL}}$.

tial perturbations is to cause $\tau_{\mathrm{NL}}$ to level off. A large enough perturbation (e.g. $\left|\delta_{\min }\right| \sim 10^{-15}$ for the mass term) allows more freedom in choosing the end of the observation window since $\tau_{\mathrm{NL}}$ no longer saturates the $10^{8}$ bound. However, we are limited in the size of the perturbation. In this model, tuning the $\phi^{2}$ term by more than $10^{-7}$ suppresses the non-Gaussianities beyond the Planck satellite's resolution. Table I gives the maximum deviation allowed for each coefficient in the potential. In order to produce an observable $\tau_{\mathrm{NL}}$ for this model, we conclude that the potential must be fine-tuned to one part in $10^{7}$.

TABLE I. Coefficient tuning in $V$ (i.e. $c \phi^{n} \rightarrow c(1+\delta) \phi^{n}$ ) for which $\left|\tau_{\mathrm{NL}}\right|$ levels off at $\sim 500$.

\begin{tabular}{ll}
\hline \hline Coefficient & $\delta_{\max }$ \\
\hline$\phi^{2}$ & $10^{-7}$ \\
$\phi^{0}$ & $10^{-4}$ \\
\hline \hline
\end{tabular}

\section{B. Log model}

The parameter choices made in the above example are by no means unique. To illustrate the diversity of possible models, we switch to the log model and consider a $\phi$-dependent speed limit:

$$
A=2, \quad B=1, \quad f=\frac{6}{m^{4}}\left(\frac{M_{P}}{\phi}\right)^{2} .
$$

In this case, the constant of integration occurring in Eq. (30) is not just a redefinition of $\phi$ and gives some freedom in defining $V$. We shall choose

$$
V=m^{4}\left[\frac{1}{4}\left(\frac{\phi}{M_{P}}\right)^{4}+\frac{2}{3}\left(\frac{\phi}{M_{P}}\right)^{2}+1\right] .
$$

For this model,

$$
\begin{gathered}
\epsilon=\frac{\left(\frac{\phi}{M_{P}}\right)^{2}}{\left[\frac{1}{2}\left(\frac{\phi}{M_{P}}\right)^{2}+1\right]^{2}}, \\
\Delta_{\mathcal{R}}^{2}=\frac{1}{24 \pi^{2}}\left(\frac{m}{M_{P}}\right)^{4}\left[\frac{1}{2}\left(\frac{\phi}{M_{P}}\right)^{2}+1\right]^{4}\left(\frac{M_{P}}{\phi}\right)^{2} \\
=2.45 \times 10^{-9}, \\
N_{e}=\frac{1}{4}\left[\left(\frac{\phi_{i}}{M_{P}}\right)^{2}-\left(\frac{\phi_{f}}{M_{P}}\right)^{2}\right]+\log \left(\frac{\phi_{i}}{\phi_{f}}\right) .
\end{gathered}
$$

Our constraints set $\phi_{0} \sim 20 M_{P}$ and $m \sim 6 \times 10^{-4} M_{P}$.

For $\phi(0)=20 M_{P}$ and $\dot{\phi}(0)=0$, the results are shown in Fig. 3. We see the main differences from the previous example are a speed limit that depends on $\phi$ and, since we are using the $\log$ model, an $f_{\mathrm{NL}}$ that slowly diverges as $\varepsilon \rightarrow$ 0 . Even so, $f_{\mathrm{NL}}$ is still predicted to be unobservably small:

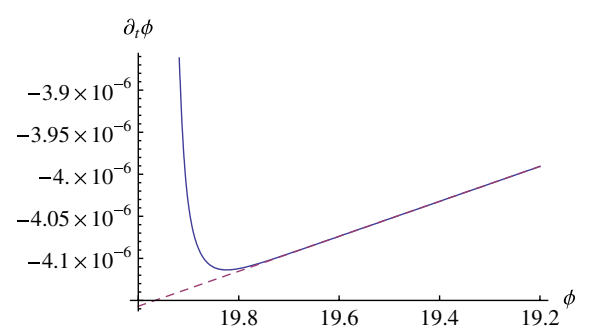

(a)

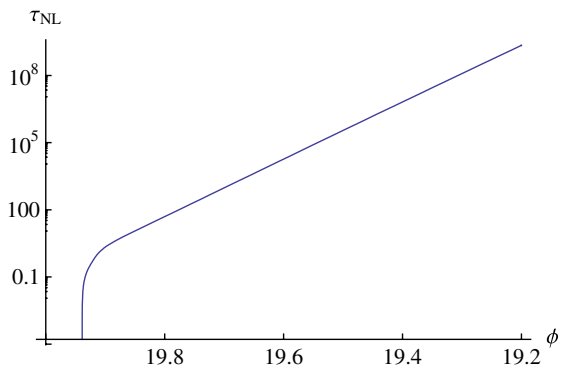

(c)

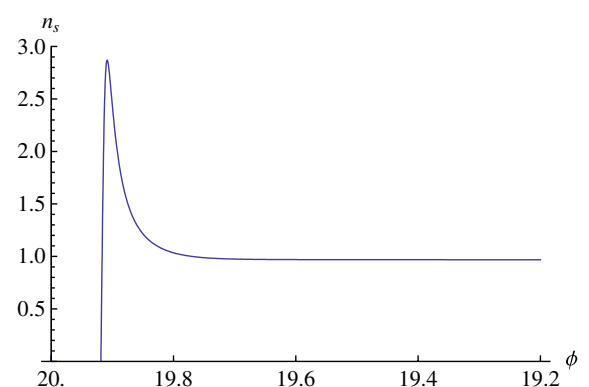

(b)

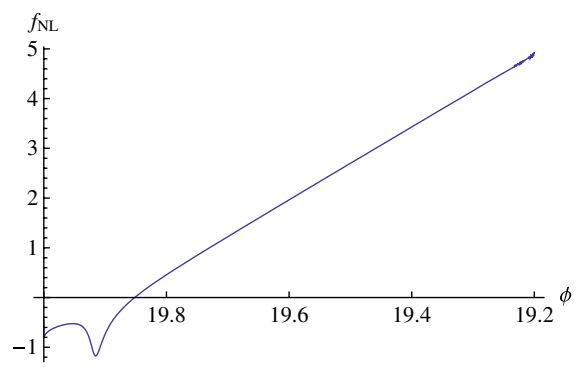

(d)

FIG. 3 (color online). (a) $\dot{\phi}$ (solid line) plotted with speed limit of $-\sqrt{\frac{2}{f}}$ (dashed line). (b) Spectral index $n_{s}$. (c) $\tau_{\mathrm{NL}}$. (d) $f_{\mathrm{NL}}$. 
TABLE II. Coefficient tuning in $V$ (i.e. $\left.c \phi^{n} \rightarrow c(1+\delta) \phi^{n}\right)$ for which $\left|\tau_{\mathrm{NL}}\right|$ levels off at $\sim 500$.

\begin{tabular}{lc}
\hline \hline Coefficient & $\delta_{\max }$ \\
\hline$\phi^{4}$ & $10^{-4}$ \\
$\phi^{2}$ & 1 \\
$\phi^{0}$ & 10 \\
\hline \hline
\end{tabular}

for $\tau_{\mathrm{NL}}$ at its bound of $10^{8}, f_{\mathrm{NL}} \sim 5$. For exact $V, \tau_{\mathrm{NL}}$ grows even faster than before; in this model, the possible observation window is only about 4 e-folds in duration. To obtain an acceptable theory, we again consider perturbations to $V$. Sufficiently large perturbations fix the problem, causing $\tau_{\mathrm{NL}}$ to level off below $10^{8}\left(\left|\delta_{\min }\right| \sim 10^{-9}\right.$ for the quartic term). The maximum deviation that still produces an observable $\tau_{\mathrm{NL}}$ is shown for each coefficient of $V$ in Table II. As predicted at the end of Sec. IV, the log model is less restrictive: the potential must be fine-tuned only to one part in $10^{4}$.

\section{CONCLUSION}

We have constructed several single-field models of inflation that satisfy current observational constraints and produce a large trispectrum and a small bispectrum. However, these features come at a cost. To generate the desired non-Gaussianities, we are forced to include an unmotivated nonstandard kinetic term in the Lagrangian. The potential cannot be arbitrary, thus introducing a degree of fine-tuning into the theory. Of the models considered, the best cases allow a tuning of order $10^{-4}$. Larger modifications result in a trispectrum unobservable by the Planck satellite. These two features-the strange kinetic term and the fine-tuned potential-seem to be general requirements for a single-field model predicting large $\tau_{\mathrm{NL}}$ and small $f_{\mathrm{NL}}$. If Planck does indeed observe a trispectrum but no bispectrum, we conclude that these undesirable attributes tend to disfavor single-field inflation. More consideration should instead be given to multiplefield models such as the curvaton mechanism, which can more naturally produce these kinds of non-Gaussianities.

\section{ACKNOWLEDGMENTS}

We thank Gary Shiu for correspondence regarding Ref. [32]. This work was supported in part by the U.S. Department of Energy (DOE) under the cooperative research agreement No. DE-FG02-92ER40701. K. L. was also supported by the Sherman Fairchild Foundation.
[1] A. H. Guth, Phys. Rev. D 23, 347 (1981).

[2] A. D. Linde, Phys. Lett. B 108, 389 (1982).

[3] A. Albrecht and P. J. Steinhardt, Phys. Rev. Lett. 48, 1220 (1982).

[4] V.F. Mukhanov, H. A. Feldman, and R. H. Brandenberger, Phys. Rep. 215, 203 (1992).

[5] A. R. Liddle and D. H. Lyth, Phys. Rep. 231, 1 (1993).

[6] J. M. Bardeen, P. J. Steinhardt, and M. S. Turner, Phys. Rev. D 28, 679 (1983).

[7] J. M. Maldacena, J. High Energy Phys. 05 (2003) 013.

[8] V. Acquaviva, N. Bartolo, S. Matarrese, and A. Riotto, Nucl. Phys. B667, 119 (2003).

[9] N. Bartolo, E. Komatsu, S. Matarrese, and A. Riotto, Phys. Rep. 402, 103 (2004).

[10] E. Silverstein and D. Tong, Phys. Rev. D 70, 103505 (2004).

[11] M. Alishahiha, E. Silverstein, and D. Tong, Phys. Rev. D 70, 123505 (2004).

[12] C. Armendariz-Picon, T. Damour, and V.F. Mukhanov, Phys. Lett. B 458, 209 (1999).

[13] J. Garriga and V.F. Mukhanov, Phys. Lett. B 458, 219 (1999).

[14] E. Komatsu and D. N. Spergel, Phys. Rev. D 63, 063002 (2001).

[15] N. Kogo and E. Komatsu, Phys. Rev. D 73, 083007 (2006).

[16] T. J. Allen, B. Grinstein, and M. B. Wise, Phys. Lett. B 197, 66 (1987).
[17] K. Enqvist and T. Takahashi, J. Cosmol. Astropart. Phys. 09 (2008) 012.

[18] M. Sasaki, J. Valiviita, and D. Wands, Phys. Rev. D 74, 103003 (2006).

[19] C. T. Byrnes, M. Sasaki, and D. Wands, Phys. Rev. D 74, 123519 (2006).

[20] J. P. Bruneton and G. Esposito-Farese, Phys. Rev. D 76, 124012 (2007); 76, 129902(E) (2007).

[21] R. Bean, D. J. H. Chung, and G. Geshnizjani, Phys. Rev. D 78, 023517 (2008).

[22] D. Babich, P. Creminelli, and M. Zaldarriaga, J. Cosmol. Astropart. Phys. 08 (2004) 009.

[23] P. Creminelli, L. Senatore, M. Zaldarriaga, and M. Tegmark, J. Cosmol. Astropart. Phys. 03 (2007) 005.

[24] L. Boubekeur and D. H. Lyth, Phys. Rev. D 73, 021301 (2006).

[25] K. M. Smith, L. Senatore, and M. Zaldarriaga, arXiv:0901.2572.

[26] E. Komatsu et al. (WMAP Collaboration), Astrophys. J. Suppl. Ser. 180, 330 (2009).

[27] K. M. Smith and M. Zaldarriaga, arXiv:astro-ph/0612571.

[28] N. Bartolo and A. Riotto, J. Cosmol. Astropart. Phys. 03 (2009) 017.

[29] X. Chen, M. x. Huang, S. Kachru, and G. Shiu, J. Cosmol. Astropart. Phys. 01 (2007) 002.

[30] D. Seery and J. E. Lidsey, J. Cosmol. Astropart. Phys. 06 (2005) 003. 
[31] F. Arroja and K. Koyama, Phys. Rev. D 77, 083517 (2008).

[32] X. Chen, M.x. Huang, and G. Shiu, Phys. Rev. D 74, 121301 (2006).
[33] D. Seery, J.E. Lidsey, and M.S. Sloth, J. Cosmol. Astropart. Phys. 01 (2007) 027.

[34] D. Seery, M.S. Sloth, and F. Vernizzi, J. Cosmol. Astropart. Phys. 03 (2009) 018. 